Original article

\title{
A simple clinical method for predicting the benefit of prone vs. supine positioning in reducing heart exposure during left breast radiotherapy
}

\author{
Zsuzsanna Kahán ${ }^{\mathrm{a}, *}$, Ferenc Rárosi ${ }^{\mathrm{b}}$, Szilvia Gaál ${ }^{\mathrm{a}}$, Adrienn Cserháti ${ }^{\mathrm{a}}$, Krisztina Boda ${ }^{\mathrm{b}}$, Barbara Darázs a \\ Renáta Kószó ${ }^{\mathrm{a}}$, Ferenc Lakosi ${ }^{c, d}$, Ákos Gulybán ${ }^{\mathrm{c}, \mathrm{e}}$, Philippe A. Coucke ${ }^{\mathrm{c}}$, Zoltán Varga ${ }^{\mathrm{a}}$

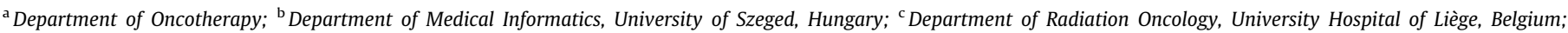 \\ ${ }^{\mathrm{d}}$ Institute of Diagnostic Imaging and Radiation Oncology, Health Center, Kaposvár University, Hungary; ${ }^{\mathrm{e}}$ Radiation Oncology Department, Europe Hospitals Brussels, Belgium
}

\section{A R T I C L E I N F O}

Article history:

Received 26 April 2017

Received in revised form 20 December 2017

Accepted 22 December 2017

Available online $\mathrm{xxxx}$

\section{Keywords:}

Breast radiotherapy

Clinical tool

Heart protection

Individual positioning

LAD protection

\begin{abstract}
A B S T R A C T
Background and purpose: The benefit of reduced radiation heart exposure in the prone vs. supine position individually differs. In this prospective cohort study, the goal was to develop a simple method for the operation of a validated model for the prediction of preferable treatment position during left breast radiotherapy.

Material and methods: In 100 cases, a single CT slice was utilized for the collection of the needed patientspecific data (in addition to body mass index, the distance of the LAD from the chest wall and the area of the heart included in the radiation fields at the middle of the heart in the supine position). Outcome was analyzed in relation to the full CT series acquired in both positions and dosimetric data.

Results: Great consistency was found between the tested and original method regarding sensitivity and specificity. The prioritization of LAD dose, and the use of heart dose and position-specific dose constraints as safety measures ensure sensitivity and specificity values of $82.8 \%$ and $87.3 \%$, respectively. In an additional "routine clinical practice" series of 60 patients the new method seemed feasible in routine clinical practice. External testing on a 28-case series indicated similar accuracy.

Conclusion: We consider this simple clinical tool appropriate for assisting individual positioning aiming at maximum heart protection during left breast irradiation.
\end{abstract}

(C) 2018 Elsevier B.V. All rights reserved. Radiotherapy and Oncology xxx (2018) xxx-xxx
Radiotherapy is an essential component of the management of early breast cancer. The outcome in most cases is favorable, the majority of the affected patients become long survivors. Breast radiotherapy, however, may increase the risk of non-breast cancer-related morbidities, among which heart diseases rank the first $[1,2]$. Radiation-induced heart damage clearly depends on the dose exposed to its different structures $(3,4)$. While older radiotherapy practices caused more significant late hazards, heightened awareness and the use of current technical developments make this danger much lower $[1,4,5]$. Although the application of modern radiotherapy planning and delivery significantly improves the control of radiation dose, in many cases a part of the heart, and especially the left anterior descending artery (LAD) located to its anterior surface still receive a dose sufficient to cause longterm adverse effects. Radiogenic diffuse myocardium damage including microvasculature abnormalities, degenerative cardiomyocyte and interstitial fibrotic changes may be controlled if not

* Corresponding author at: Department of Oncotherapy, University of Szeged, Korányi fasor 12, H-6720 Szeged, Hungary.

E-mail address: kahan.zsuzsanna@med.u-szeged.hu (Z. Kahán). extensive, but the damage of the macrovasculature indistinguishable from coronary arteriosclerosis due to other causes more likely lead to a fatal outcome $[3,6-8]$. The exposure of the heart and the LAD are related [9-11], and irradiation-related cardiac morbidity and mortality are considered to be consequences of late manifesting coronary artery damage. Hence the verification and control of the dose to the LAD, is of prime importance $[8,9,11,12]$.

With the aim of cardiac dose sparing and avoidance, numerous new methods have been developed $[4,5]$. These include the breathholding techniques, prone positioning (both operate by separating the heart and the radiation fields), IMRT, proton irradiation or the reduction in the volume to be irradiated, partial breast irradiation (PBI). A significant increase in the number of clinical studies [11-20], and a recent survey on clinical practice [21] suggest that prone positioning has become an alternative of conventional supine positioning in some centers. Prone positioning always provides dramatic reduction in the ipsilateral lung dose, and in many cases significantly reduces heart exposure, too. A potential disadvantage is inferior repositioning accuracy, which may be improved with experience [18] or may be compensated by online daily correction [12,22]. 
Prone positioning was first invented for the irradiation of largebreasted women $[23,24]$. Indeed, since gravity pulls the breast away from the chest wall, the geometry of a pendulous breast and the tangential irradiation fields gets advantageous in the prone position [12]. Taking the overall population of breast cancer patients, however, prone positioning has such effect in $77-87 \%$ of cases only $[11,14,15,19]$. As a consequence, the positiondependent dose to the LAD or heart also individually differs $[11,19,20]$. Different approaches exist for selecting the optimal position in left breast cancer cases. Kirby et al. found that a PTV $>1000 \mathrm{~cm}^{3}$ favors prone positioning [11]. Zhao et al. developed a two-step decision-analysis algorithm that, based on the anatomical features detected on a prone $\mathrm{CT}$ series, classified patients to prone radiotherapy or to a second $\mathrm{CT}$ in the supine position for comparison [25]. We have demonstrated that a statistical model utilizing 3 anatomical determinants (the body mass index [BMI], the distance of the LAD from the chest wall and the area of the heart included in the radiation fields at the middle of the heart in the supine position) of the patient gives accurate estimates on the benefit of one specific position over the other by means of LAD or heart doses [19]. Here we report on an original method for providing the necessary patient-specific data based on a single CT slice image representing the middle of the heart. In this prospective study, following the validation of the clinical tool, also its routine use has been tested on a separate series of cases.

\section{Patients and methods}

The study was approved by the Institutional Review Board of the University of Szeged, and all the enrolled patients gave their written informed consent to participation. Eligible patients needed postoperative left breast radiotherapy.

\section{Outline of the study}

First, a single CT slice image at the middle of the heart (reference plane, $\left.P_{\text {ref }}\right)$ was acquired with the help of an AP scout view in the supine position (Fig. 1A). On that CT scan, the shortest distance between the anterior surface of the LAD and the chest wall $\left(D_{\text {med }}\right)$ and the area of the heart $\left(A_{\text {heart }}\right)$ included in the radiation fields were measured after placing a straight line between the border of the ipsilateral latissimus dorsi muscle and the lateral edge of the sternum (Fig. 1B); these data (representing the topography of the heart) were introduced to the calculator together with the patient's BMI (which correlated with the volumes of the breast and heart) as previously described in detail [19]. The calculator based on a validated statistical model provided the estimated LAD and heart dose differences in the prone vs. supine position of the individual patient. In the first validation set of 100 patients,

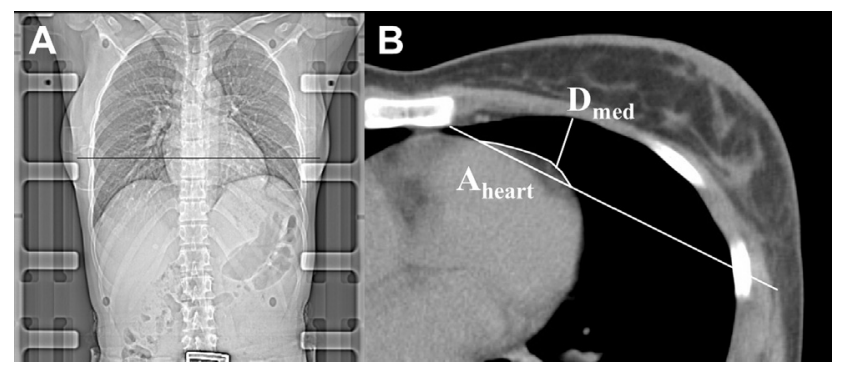

Fig. 1. The simple clinical tool generates patient-specific data to predict the benefit of prone positioning. After selecting the reference plane $\left(P_{\text {ref }}\right)$ at the middle of the heart on the AP scout view (A), a single CT slice is acquired for the measurement of those determinants ( $D_{\text {med }}$ and $A_{\text {heart }}$ ) (B) which operate the calculator to provide estimates of the doses to the LAD or heart.
CT series were acquired in both the supine position and prone position. Conformal radiation treatment plans were generated in both positions using conventional $6 \mathrm{MV}$ tangential photon fields set up isocentrically and median 2 (1-3) individually weighted 6/15 MV segmental fields superimposed on the tangential fields using a multileaf collimator as described $[18,19]$. Wedges were used in almost all supine radiation plans. A mean dose to the PTV of 50 Gy (25 fractions) and a uniform distribution $(-5 \%+7 \%)$ of the prescribed dose to $95 \%$ of the PTV, were aimed at. The consistency of all contouring activities had been ensured by a chief radiation oncologist (ZK) and an experienced radiologist (AC) [26]. Equivalent heart and LAD volume contouring in either setup was ensured by one author (ZK). In the next "routine clinical practice" set of 60 patients, the acquisition of a single series of CT images according to the suggestion of the calculator was aimed at, and a second CT series was taken only if any of the dose constraints approved for the specific position were not reached in the position suggested by the calculator. In this series of patients' dose constraints were specified on the basis of previously recorded data. The upper range limits of the $90 \%$ percentile of dosimetry data in the preferred position were the following: mean LAD dose $\left[\mathrm{MD}_{\mathrm{LAD}}\right]: 12.9 \mathrm{~Gy}$ and $12.5 \mathrm{~Gy}$, $V_{25 \text { Gyheart }}: 2.4 \%$ and $4.7 \%$, in the prone position and supine position, respectively. In true discordant cases, our strategy for selecting treatment position was to consider the LAD dose as a primary decisive factor.

In the validation set, data on LAD and heart dose differences between the two treatment positions were extracted from the planning system and estimated by the calculator, whereas in the "routine clinical practice" series only the estimated dose differences were available. Analyses were performed on 1 . the equivalence of the $P_{\text {ref }}$ with the median plane of the full series of CT scans acquired in the supine position $\left(P_{\text {med }}\right)$ and 2 . the effect of plane miss on the patient-related determinants and choice of preferable position. The sensitivity and specificity of this simple clinical method were evaluated based on the dosimetry data obtained using the topogram for selecting the position $(n=100)$. In the "routine clinical practice" series, the acceptability of the position as predicted by the calculator, the LAD and heart doses achieved without taking 2 CT series, and the need of performing a second CT series and changing position or irradiation technique were analyzed.

\section{External testing}

The supine and prone CT series and supine topogram of patients included in the study "Individualized positioning for maximum heart and index breast protection during breast irradiation: comparative study between Prone and Supine (Approval: 26/09/2013, B707201318246) were retrospectively used for independent testing. The protocol of patient positioning, delineation and radiation treatment planning has been described [27].

First, $P_{\text {ref }}$ was selected on the topogram. Then, the predictors BMI, $D_{\text {med }}, A_{\text {heart }}$ as measured in $P_{\text {ref }}$ were introduced to the calculator. As a second step, $D_{\text {med }}, A_{\text {heart }}$ were also measured in $P_{\text {med. }}$ LAD and heart dose differences between the two treatment positions extracted from the planning system and estimated by the calculator were analyzed. Finally, the correctness of $P_{\text {ref }}$ was evaluated.

\section{Statistical methods}

The calculator had been developed based on linear regression models utilizing the patients' anatomical features, with $\triangle \mathrm{MD}_{\mathrm{LAD}}$ and $\Delta V_{25 \text { Gyheart }}$ as dependent variables [19]. With a single cut-off point, a case was classified to prone positioning when the predicted value exceeded that value. Thresholds were optimized based on sensitivity and specificity as calculated from previous 
Table 1

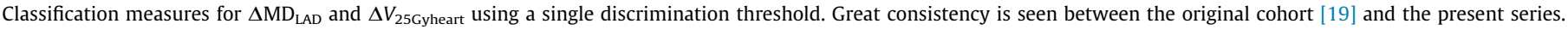

\begin{tabular}{|c|c|c|c|c|c|}
\hline & \multirow[b]{2}{*}{ Cut-off point } & \multicolumn{2}{|c|}{ Original method (double CT method, $n=83$ ) } & \multicolumn{2}{|c|}{ Simple tool (single CT method, $n=100$ ) } \\
\hline & & Sensitivity (\%) & Specificity (\%) & Sensitivity (\%) & Specificity (\%) \\
\hline \multirow[t]{9}{*}{$\Delta \mathrm{MD}_{\mathrm{LAD}}(\mathrm{Gy})$} & -0.6 & 66.6 & 91.1 & 72.4 & 91.5 \\
\hline & -0.3 & 70.8 & 90.7 & 75.9 & 91.5 \\
\hline & 0 & 74.4 & 90.0 & 75.9 & 91.5 \\
\hline & 0.3 & 77.7 & 88.9 & 79.3 & 88.7 \\
\hline & 0.6 & 80.7 & 87.5 & 82.8 & 87.3 \\
\hline & 0.9 & 83.4 & 86.0 & 82.8 & 83.1 \\
\hline & 1.2 & 85.4 & 83.6 & 86.2 & 81.7 \\
\hline & 1.5 & 86.5 & 81.7 & 86.2 & 77.5 \\
\hline & 1.8 & 86.8 & 79.9 & 93.1 & 76.1 \\
\hline \multirow[t]{8}{*}{$\Delta V_{25 \text { Gyheart }}(\%)$} & 0 & 47.9 & 89.7 & 50 & 90.8 \\
\hline & 0.25 & 56.2 & 88.8 & 58.3 & 89.5 \\
\hline & 0.50 & 63.2 & 85.9 & 64 & 88 \\
\hline & 0.75 & 72.4 & 82.4 & 68 & 85.3 \\
\hline & 1 & 78.8 & 77.7 & 80 & 85.3 \\
\hline & 1.25 & 84.0 & 74.0 & 84 & 81.3 \\
\hline & 1.50 & 87.4 & 77.0 & 92 & 78.6 \\
\hline & 1.75 & 89.9 & 62.1 & 96 & 74.6 \\
\hline
\end{tabular}

Table 2

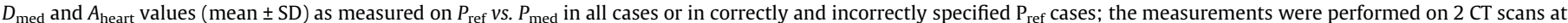
the middle of the heart either identified with the help of an A-P scout view $\left(\mathrm{P}_{\text {ref }}\right)$ or selected from a full CT series $\left(P_{\text {med }}\right)$.

\begin{tabular}{|c|c|c|c|c|c|c|}
\hline & \multicolumn{2}{|c|}{ All cases $(n=100)$} & \multicolumn{2}{|c|}{ Correct plane $(n=55)$} & \multicolumn{2}{|c|}{ Plane miss $(n=45)$} \\
\hline & $P_{\text {ref }}$ & $P_{\text {med }}$ & $P_{\text {ref }}$ & $P_{\text {med }}$ & $P_{\text {ref }}$ & $P_{\text {med }}$ \\
\hline$D_{\text {median }}(\mathrm{cm})$ & $1.27 \pm 0.59$ & $1.25 \pm 0.67$ & $1.35 \pm 0.55$ & $1.17 \pm 0.63$ & $1.18 \pm 0.63$ & $1.34 \pm 0.71$ \\
\hline$A_{\text {heart }}\left(\mathrm{mm}^{2}\right)$ & $768.8 \pm 487.4$ & $671.6 \pm 450.1$ & $730.7 \pm 537.4$ & $721.5 \pm 511.2$ & $815.4 \pm 419.5$ & $610.5 \pm 358.1$ \\
\hline
\end{tabular}

[19] and present data (Table 1). Sensitivity and specificity were calculated with supine positioning as positive determinant in the model. For $\Delta \mathrm{MD}_{\mathrm{LAD}}$ a threshold of $0.6 \mathrm{~Gy}$, and for $\Delta V_{25 \mathrm{Gyheart}}$ a cut-off point of $1.0 \%$ were chosen. In the definition of the cut-off points, a sensitivity of $80 \%$ at the minimum and the maximum achievable value of specificity was required.

LAD and heart dose constraints achievable by selecting the preferable position were specified by percentage estimation. Statistical analysis was performed with SPSS 22.0 for Windows.

\section{Results}

\section{Validation set}

In 55/100 cases, $P_{\text {ref }}$ was the same as $P_{\text {med }}$ while in 28 and 17 cases, $P_{\text {ref }}$ and $P_{\text {med }}$ differed by 1 or more planes, respectively. More among the incorrectly defined $P_{\text {ref }}$ cases were shifted toward the caudal than the cranial direction. This resulted in smaller mean $D_{\text {med }}$ and larger mean $A_{\text {heart }}$ values among the plane miss cases overall (Table 2). Within the whole series, no change in the frequency of plane misses could be detected by time. Incongruency among $\Delta M_{\mathrm{LAD}}$ and $\Delta V_{25 \mathrm{Gyhear}}$ in the supine and prone position as predicted by the calculator on the basis of $P_{\text {ref }} v s$. $P_{\text {med }}$ data, was present in 14 and 18 of the cases, respectively; these were all of small numerical values (Fig. 2A, B). When the LAD and heart dose differences predicted by the calculator based on the $P_{\text {ref }}$ values were compared with the original dosimetric data from plans generated in both positions, the suggestion proved invalid in 14 $\left(\mathrm{MD}_{\mathrm{LAD}}\right)$ and 16 ( $\left.V_{25 \mathrm{Gyheart}}\right)$ cases (Fig. 2C, D). We have compared the sensitivity and specificity of $\Delta \mathrm{MD}_{\mathrm{LAD}}$ and $\Delta V_{25 \mathrm{Gyheart}}$ provided by the simple method based on a single CT scan with that of the original method that indicated high consistency [19] (Table 1). Based on these findings, the cut-off values of $0.6 \mathrm{~Gy}\left(\Delta \mathrm{MD}_{\mathrm{LAD}}\right)$ and $1.0 \%\left(\Delta V_{25 \text { Gyheart }}\right)$ have been selected for further analyses and practice.
Next, the concordance of calculator-predicted treatment position based on $\Delta \mathrm{MD}_{\mathrm{LAD}} v s . \Delta V_{25 \mathrm{Gyheart}}$ and the need for intervention were analyzed in the validation set. In 28 supine-predicted cases and 64 prone-predicted cases, the same treatment position was suggested by both measures (Table 3 ). Among the 28 supinepredicted cases in 2 , the radiotherapy plan revealed that $\mathrm{MD}_{\mathrm{LAD}}$ $>12.5 \mathrm{~Gy}$, but only 1 could be improved by changing the treatment position. Among the 64 prone-predicted cases in 8 , the $\mathrm{MD}_{\mathrm{LAD}}$ exceeded the dose constraint of $12.9 \mathrm{~Gy}$; only 3 plans could be improved by applying the supine position. Among the discordant cases, $\triangle \mathrm{MD}_{\mathrm{LAD}}$ suggested prone position in 3 and supine position in 5 cases; in both groups in a single case each could the LAD dose be improved by changing the treatment position. In altogether 7 cases, a different intervention (IMRT) had to be applied (Table 3).

\section{"Routine clinical practice" set}

In the "routine clinical practice" series of 60 patients, the new method proved feasible. All patients received treatment in the position suggested by the calculator except one, who had to receive a second $\mathrm{CT}$ in the other position due to unacceptable LAD dose. The other patients had $\mathrm{MD}_{\mathrm{LAD}}$ and $V_{25 \text { Gyheart values well below }}$ the predefined dose limits, and these were similar to the values calculated in the validation set (Table 4).

\section{External testing}

In a series of 28 breast cancer patients from Liege, the predictors BMI, $D_{\text {med }}$ and $A_{\text {heart }}$ significantly differed from the same parameters among the patients from Szeged. In 18/28 cases, $P_{\text {ref }}$ was equal or close to $P_{\text {med }}(\leq 6 \mathrm{~mm})$, while in 10 , cases $P_{\text {ref }}$ varied from $P_{\text {med }}$ by 9-16 mm. Comparing the calculator-provided dose differences with the treatment planning data, favored treatment position was correct in 24/28 (accuracy: $85.7 \%$ ) and 23/28 (accuracy: $82.1 \%$ ) cases taking into account the LAD and heart doses, 


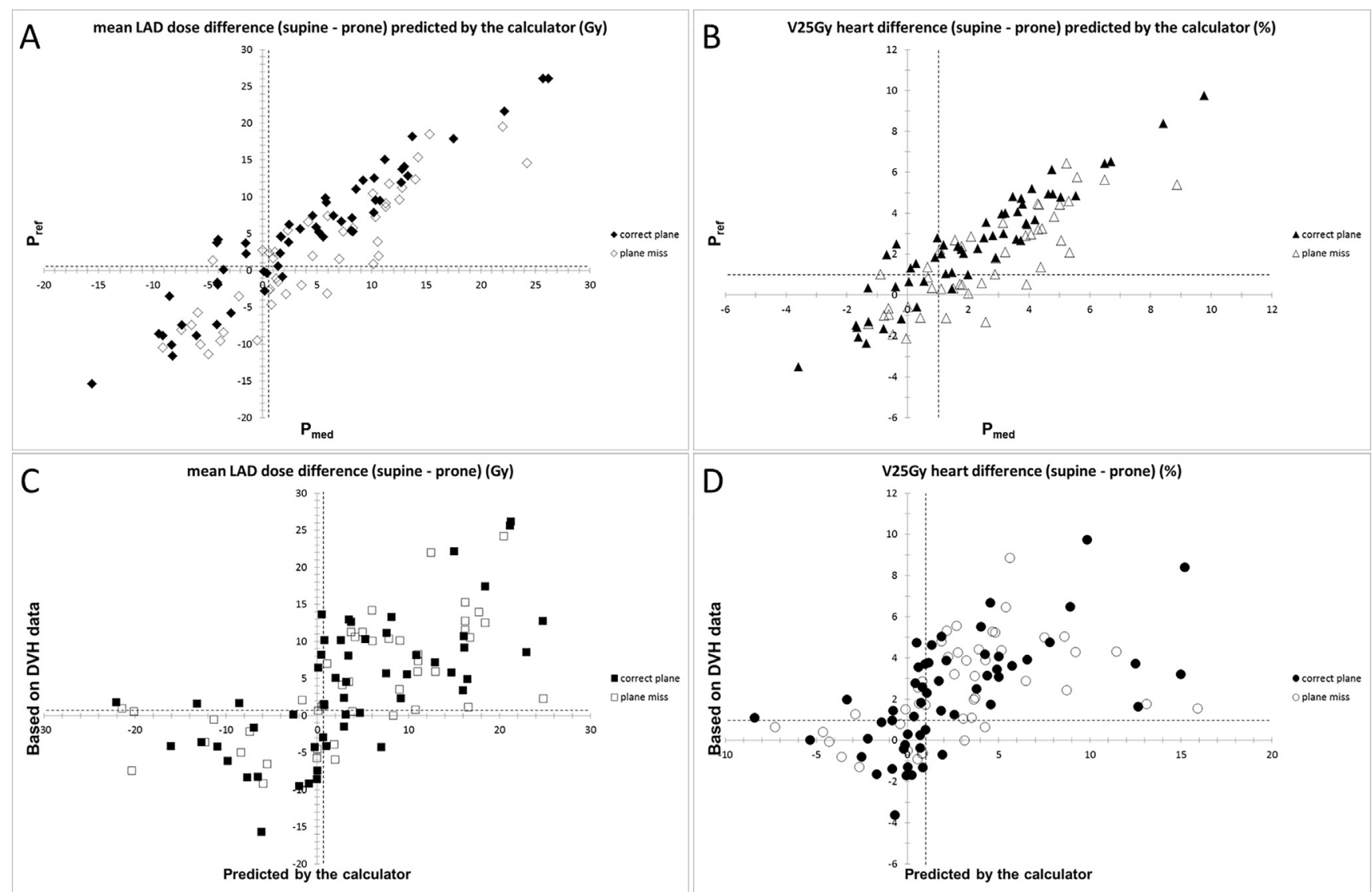

Fig. 2. Calculator suggestion of LAD (A) and heart (B) dose differences by the input of $D_{\text {med }}$ and $A_{\text {heart }}$ based on $P_{\text {ref }} v s$. $P_{\text {med }}$; LAD (C) and heart (D) doses according to the estimation of the simple clinical method based on a single CT scan vs. DVH data extracted from the planning system $(n=100)$. Dashed lines indicate the cut-off values of 0.6 Gy $\left(\Delta \mathrm{MD}_{\mathrm{LAD}}\right)$ and $1.0 \%\left(\Delta V_{25 \text { Gyheart }}\right)$ specified by sensitivity and specificity values.

Table 3

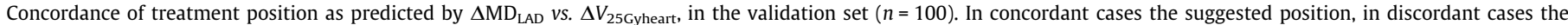
position suggested by $\triangle \mathrm{MD}_{\mathrm{LAD}}$ was applied unless the dose constraints were exceeded; in such cases the other treatment position or alternative techniques may be tested.

\begin{tabular}{|c|c|c|c|c|c|c|c|c|c|}
\hline & & \multicolumn{8}{|c|}{$\Delta V_{25 \text { Gyheart }}$} \\
\hline & & \multicolumn{4}{|c|}{ Supine } & \multicolumn{4}{|c|}{ Prone } \\
\hline & & All & $\mathrm{MD}_{\mathrm{LAD}}>12.5 \mathrm{~Gy}$ & Change position & Other intervention & All & $\mathrm{MD}_{\mathrm{LAD}}>12.9 \mathrm{~Gy}$ & Change position & Other intervention \\
\hline \multirow[t]{2}{*}{$\Delta \mathrm{MD}_{\mathrm{LAD}}$} & Supine & 28 & 2 & $1 / 2$ & $1 / 2$ & 5 & $1 / 5$ & $1 / 1$ & - \\
\hline & Prone & 3 & $2 / 3$ & $1 / 2$ & $1 / 2$ & 64 & $8 / 64$ & $3 / 8$ & $5 / 8$ \\
\hline
\end{tabular}

Table 4

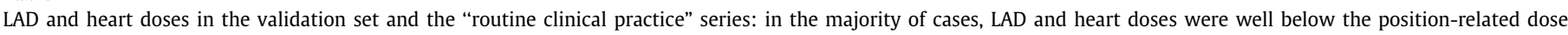
constraints; for those patients who had higher than accepted doses, an alternative technique had to be applied.

\begin{tabular}{|c|c|c|c|c|c|c|c|c|c|c|}
\hline & \multirow[t]{2}{*}{ Treatment position } & \multirow[t]{2}{*}{$n(\%)$} & \multicolumn{4}{|c|}{ Mean LAD dose (Gy) } & \multicolumn{4}{|c|}{$V_{25 G \text { yheart }}(\%)$} \\
\hline & & & Mean & SD & Min & Max & Mean & SD & Min & Max \\
\hline \multirow[t]{2}{*}{ Validation series } & Prone & $67(67.0)$ & 6.55 & 6.03 & 1.70 & 26.66 & 1.16 & 2.24 & 0.0 & 8.75 \\
\hline & Supine & $33(33.0)$ & 6.90 & 3.86 & 1.71 & 13.73 & 1.54 & 1.38 & 0.0 & 4.77 \\
\hline \multirow[t]{2}{*}{ "Routine clinical practice" series } & Prone & $47(78.3)$ & 6.58 & 2.29 & 1.95 & 11.24 & 0.86 & 0.57 & 0.1 & 2.67 \\
\hline & Supine & $13(21.7)$ & 7.35 & 3.05 & 2.54 & 15.85 & 1.15 & 0.95 & 0.21 & 3.57 \\
\hline
\end{tabular}

respectively. Sensitivity and specificity of $\triangle \mathrm{MD}_{\mathrm{LAD}}$ was $83.3 \%$ and $86.4 \%$, respectively, whereas sensitivity and specificity of $\Delta V_{25 \text { Gyheart }}$ was $100.0 \%$ and $80.0 \%$, respectively.

\section{Discussion}

According to the present study and others $[11,14,15,19,20]$, in about $20 \%$ of the cases, prone positioning during left breast radio- therapy increases the dose to the LAD or the heart. To estimate and select the preferable positioning mode, supine CT seems the best approach to consider the patient's anatomical determinants. We have shown that a single CT scan at the middle of the heart may replace a whole CT series by providing consistent anatomical data thus avoiding extra radiation exposure to the patient and work load to the staff. Based on the outcome of the external implementation of the method on an independent case series, we recommend its use after local testing. 
Our validated statistical model for predicting the preferable treatment position utilizes 3 specific measures, and seems the most complex predictive tool for this purpose in the literature [19]. In other studies, the in-field heart volume $[16,17,25]$ and most frequently the size of the breast $[4,11]$ have been used for selection. An increased BMI has also been related to larger heart doses [28] or consequential radiation cardiac morbidity [29], but its role in predicting benefit of prone positioning may be refined by the use of other patient-related parameters [19]. We consider the BMI in our calculator as a stable parameter while there is potential uncertainty in the specification of $P_{\text {ref }}$ or imprecision in the actual measurement of $D_{\text {median }}$ or $A_{\text {heart }}$ on a given image. Nevertheless, detailed analysis indicates that accidental imprecision does not significantly influence final prediction (data not shown). The dose constraints optimized by individual positioning provides additional safety in practice. Despite the lack of full equivalence of the data extracted from the original method vs. the new method, the ultimate consistency still seems to qualify the developed "simple tool" for clinical application.

External use indicated similar accuracy as the originally developed method. Despite the reassuring results on an independent series of patients in a radiotherapy center using a slightly different protocol, the utility of the reported clinical tool could be compromised by the diversity of practice in others. PTV contouring depends on repositioning accuracy and the method of treatment verification. Interfractional differences may be especially large in the prone position $[18,30]$. Lakosi et al. found population systematic error values of $4.5 / 3.9 / 3.3 \mathrm{~mm}$ in the lateral/longitudinal/vertical directions, while the random error was $5.4 / 3.8 / 2.8 \mathrm{~mm}$ [27]. Among our recent breast radiotherapy cases, the population systematic and random error in the lateral/longitudinal/vertical directions was similar in the prone position vs. supine position (3.4/2.3/2.7 $\mathrm{mm}$ and $7.8 / 4.6 / 6.9 \mathrm{~mm}$, respectively vs. $2.2 / 3.0 / 1.6$ $\mathrm{mm}$ and $6.7 / 5.5 / 4.5 \mathrm{~mm}$, respectively). Only some groups study the dose to the coronary arteries $[11,12,19,20,31-34]$. The outlining of the coronary vessels shows significant inter-observer variation that may jeopardize dose verification in the selected position [35,36]. Different approaches have been tested to improve consistency including the administration of contrast media [35-37]. Lee et al. developed a new protocol to outline the LAD region which included $96 \%$ of the LAD volume as delineated by 4 experienced radiation oncologists [37]. Significant impact was made by the implementation of specific guidelines [35-37]. Since the utility of the simple tool might be influenced by several factors, in addition to the use of institutional LAD contouring guidelines and study of inter-observer variation, we consider essential its testing before routine use. In the case of hypofractionated radiotherapy, the model parameters of the calculator should be re-estimated and the dose constraints should be re-defined.

The benefit of positioning prone vs. supine may be discordant by means of LAD and heart doses [11,19,34]. We regard the LAD dose as a surrogate indicator of radiation harm due to its proven role in late cardiac morbidity [3] and because the LAD being situated on the anterior surface of the heart is a sensitive marker of danger if the heart is at all included into radiation. Our strategy for optimization in individual cases is to consider the $\mathrm{MD}_{\mathrm{LAD}}$ as priority that is usually confirmed by the heart dose (as was true for $92 \%$ of cases in our series).

The radiation exposure of the heart may be significantly reduced by the use of respiration-guided techniques including the deep inspiration breath hold (DIBH) technique and respiratory gating. In the UK HeartSpare study, supine DIBH provided superior cardiac sparing than a free-breathing prone position in largebreasted women [12]. Interestingly, the implementation of DIBH in the prone position gave the optimal heart sparing results as compared with that in the supine position or free-breathing [33].
There are some centers that due to resource limitations prioritize high cardiac dose cases for DIBH [38]. Our tool could be used for patients either not amenable for or not having access to DIBH due to patient-specific features (cardiorespiratory problems, lack of compliance) or limited/no resources, respectively.

We think that since a linear, no-threshold association exists between the mean heart dose and coronary events [3], doses to the LAD, right coronary artery or the circumflex artery should be controlled [20]. Nevertheless, the utilization of heart dose-volume data only is a possibility if LAD contouring cannot be afforded. Since good agreement exists between the mean heart dose and $V_{25 \text { Gyheart }}$ (Rprone: 0.98 , Rsupine: 0.99 ) or $\mathrm{MD}_{\text {LAD }}$ (Rprone and Rsupine: 0.87 ) in both positions ( $p<0.001$ in all comparisons), here the presented tool could be adapted to practices which adhere to the consideration of the mean heart dose.

In summary, we have demonstrated great consistency of our method based on a validated model for the prediction of treatment position prone vs. supine with less heart exposure during left breast radiotherapy; the simplified tool presented here omits the performance of planning $\mathrm{CT}$ in both positions. Based on the results of its external testing, we truly recommend its use in centers that apply prone positioning in routine clinical practice. Due to differences in populations and radiotherapy protocols, local testing is essential.

\section{Conclusion}

We consider this simple clinical tool useful for assisting individual positioning in routine clinical practice aiming at maximum heart protection during left breast irradiation.

\section{Conflict of interest}

None declared.

\section{Acknowledgement}

This work had been supported by the VKSZ 12-1-2013-0012 project. The authors are grateful to Edit Kiss and Laura Gál for their excellent technical support.

\section{References}

[1] Clarke M, Collins R, Darby S, et al. Early Breast Cancer Trialists' Collaborative Group (EBCTCG). Effects of radiotherapy and of differences in the extent of surgery for early breast cancer on local recurrence and 15-year survival: an overview of the randomised trials. Lancet 2005:366:2087-106.

[2] McGale P, Darby SC, Hall P, et al. Incidence of heart disease in 35,000 women treated with radiotherapy for breast cancer in Denmark and Sweden. Radiother Oncol 2011:100:167-75.

[3] Darby SC, Ewertz M, McGale P, et al. Risk of ischemic heart disease in women after radiotherapy for breast cancer. N Engl J Med 2013;368:987-98.

[4] Taylor CW, Kirby AM. Cardiac side-effects from breast cancer radiotherapy. Clin Oncol (R Coll Radiol) 2015;7:621-9.

[5] Shah C, Badiyan S, Berry S, et al. Cardiac dose sparing and avoidance techniques in breast cancer radiotherapy. Radiother Oncol 2014;112:9-16.

[6] Correa CR, Litt HI, Hwang WT, Ferrari VA, Solin LJ, Harris EE. Coronary artery findings after left-sided compared with right-sided radiation treatment for early-stage breast cancer. J Clin Oncol 2007;25:3031-7.

[7] Harris EE, Correa C, Hwang WT, et al. Late cardiac mortality and morbidity in early-stage breast cancer patients after breast-conservation treatment. I Clin Oncol 2006;24:4100-6.

[8] Nilsson G, Holmberg L, Garmo H, et al. Distribution of coronary artery stenosis after radiation for breast cancer. J Clin Oncol 2012:30:380-6.

[9] Nilsson G, Witt Nyström P, Isacsson U, et al. Radiation dose distribution in coronary arteries in breast cancer radiotherapy. Acta Oncol 2016;55:959-63.

[10] Becker-Schiebe M, Stockhammer M, Hoffmann W, Wetzel F, Franz H. Does mean heart dose sufficiently reflect coronary artery exposure in left-sided breast cancer radiotherapy?: influence of respiratory gating. Strahlenther Onkol 2016;192:624-31. 
[11] Kirby AM, Evans PM, Donovan EM, Convery HM, Haviland JS, Yarnold JR. Prone versus supine positioning for whole and partial-breast radiotherapy: a comparison of non-target tissue dosimetry. Radiother Oncol 2010;96:178-84.

[12] Bartlett FR, Colgan RM, Donovan EM, et al. The UK HeartSpare Study (Stage IB): randomised comparison of a voluntary breath-hold technique and prone radiotherapy after breast conserving surgery. Radiother Oncol 2015;114:66-72.

[13] Griem KL, Fetherston P, Kuznetsova M, et al. Three-dimensional photon dosimetry: A comparison of treatment of the intact breast in the supine and prone position. Int J Radiat Oncol Biol Phys 2003;57:891-9.

[14] Lymberis SC, DeWyngaert JK, Parhar P, et al. Prospective assessment of optimal individual position (prone versus supine) for breast radiotherapy: volumetric and dosimetric correlations in 100 patients. Int J Radiat Oncol Biol Phys 2012;84:902-9.

[15] Formenti SC, DeWyngaert JK, Jozsef G, Goldberg JD. Prone vs supine positioning for breast cancer radiotherapy. JAMA 2012;308:861-3.

[16] DeWyngaert JK, Jozsef G, Mitchell J, Rosenstein B, Formenti SC. Accelerated intensity-modulated radiotherapy to breast in prone position: dosimetric results. Int J Radiat Oncol Biol Phys 2007;68:1251-9.

[17] Formenti SC, Gidea-Addeo D, Goldberg JD, et al. Phase I-II trial of prone accelerated intensity modulated radiation therapy to the breast to optimally spare normal tissue. J Clin Oncol 2007;25:2236-42.

[18] Varga Z, Hideghéty K, Mező T, Nikolényi A, Thurzó L, Kahán Z. Individual positioning: a comparative study of adjuvant breast radiotherapy in the prone versus supine position. Int J Radiat Oncol Biol Phys 2009;75:94-100.

[19] Varga Z, Cserháti A, Rárosi F, et al. Individualized positioning for maximum heart protection during breast irradiation. Acta Oncol. 2014;53:58-64.

[20] Würschmidt F, Stoltenberg S, Kretschmer M, Petersen C. Incidental dose to coronary arteries is higher in prone than in supine whole breast irradiation. A dosimetric comparison in adjuvant radiotherapy of early stage breast cancer. Strahlenther Onkol 2014;190:563-8.

[21] Dundas KL, Pogson EM, Batumalai V, et al. Australian survey on current practices for breast radiotherapy. J Med Imaging Radiat Oncol 2015;59:736-42.

[22] Lakosi F, Gulyban A, Janvary L, et al. Respiratory motion, anterior heart displacement and heart dosimetry: comparison between prone (Pr) and supine (Su) whole breast irradiation. Pathol Oncol Res 2015;21:1051-8.

[23] Merchant TE, McCormick B. Prone position breast irradiation. Int J Radiat Oncol Biol Phys 1994;30:197-203.

[24] Buijsen J, Jager JJ, Bovendeerd J, et al. Prone breast irradiation for pendulous breasts. Radiother Oncol 2007;82:337-40.

[25] Zhao X, Wong EK, Wang Y, et al. A support vector machine (SVM) for predicting preferred treatment position in radiotherapy of patients with breast cancer. Med Phys 2010;37:5341-50.
[26] Feng M, Moran JM, Koelling T, et al. Development and validation of a heart atlas to study cardiac exposure to radiation following treatment for breast cancer. Int J Radiat Oncol Biol Phys 2011;79:10-8.

[27] Lakosi F, Gulyban A, Ben-Mustapha Simoni S, et al. Feasibility evaluation of prone breast irradiation with the Sagittilt $\subset$ system including residualintrafractional error assessment. Cancer Radiother 2016;20:776-82.

[28] Evans SB, Sioshansi S, Moran MS, Hiatt J, Price LL, Wazer DE. Prevalence of poor cardiac anatomy in carcinoma of the breast treated with whole-breast radiotherapy: reconciling modern cardiac dosimetry with cardiac mortality data. Am J Clin Oncol 2012;35:587-92.

[29] Evans ES, Prosnitz RG, Yu X, et al. Impact of patient-specific factors, irradiated left ventricular volume, and treatment set-up errors on the development of myocardial perfusion defects after radiation therapy for left-sided breast cancer. Int J Radiat Oncol Biol Phys 2006;66:1125-34.

[30] Mulliez T, Gulyban A, Vercauteren T, et al. Setup accuracy for prone and supine whole breast irradiation. Strahlenther Onkol 2016;192:254-9.

[31] Aznar MC, Korreman SS, Pedersen AN, Persson GF, Josipovic M, Specht L. Evaluation of dose to cardiac structures during breast irradiation. Br J Radiol 2011:84:743-6.

[32] Mulliez T, Veldeman L, van Greveling A, et al. Hypofractionated whole breast irradiation for patients with large breasts: a randomized trial comparing prone and supine positions. Radiother Oncol 2013;108:203-8.

[33] Mulliez T, Veldeman L, Speleers B, et al. Heart dose reduction by prone deep inspiration breath hold in left-sided breast irradiation. Radiother Oncol 2015;114:79-84.

[34] Verhoeven K, Swelden C, Petillion S, et al. Breathing adapted radiation therapy in comparison with prone position to reduce the doses to the heart, left anterior descending artery and contralateral breast in whole breast radiation therapy. Pract Radiat Oncol 2014;4:123-9.

[35] Lorenzen EL, Taylor CV, Maraldo M, et al. Inter-observer variation in delineation of the heart and left anterior descending coronary artery in radiotherapy for breast cancer: a multi-centre study from Denmark and the UK. Radiother Oncol 2013;108:254-8.

[36] Duane F, Aznar MC, Bartlett F, et al. A cardiac contouring atlas for radiotherapy Radiother Oncol 2017;122:416-22.

[37] Lee J, Hua K-L, Hsu S-M, et al. Development of delineation for the left anterior descending coronary artery region in left breast cancer radiotherapy: an optimized organ at risk. Radiother Oncol 2017;122:423-30.

[38] Tanna N, McLauchlan R, Karis S, Welgemoed C, Gujral DM, Cleator SJ Assessment of upfront selection criteria to prioritise patients for breath-hold left-sided breast radiotherapy. Clin Oncol (R Coll Radiol) 2017;29:356-61. 